\title{
Variable effects of statins on glucose homeostasis parameters and their diabetogenic role. Reply to Kostapanos MS, Agouridis AP and Elisaf MS [letter]
}

\author{
Henna Cederberg ${ }^{1}$ - Markku Laakso ${ }^{1}$
}

Received: 21 May 2015 / Accepted: 29 May 2015 / Published online: 25 June 2015

(C) Springer-Verlag Berlin Heidelberg 2015

Keywords Diabetes · Insulin secretion · Insulin sensitivity and resistance $\cdot$ Statin

\begin{abstract}
Abbreviations
HMG-CoA Hydroxy-methyl-glutaryl coenzyme A

METSIM Metabolic Syndrome in Men
\end{abstract}

We thank Dr Kostapanos and collaborators for their comments on our article $[1,2]$. The authors refer to their previous small study, which included 72 hyperlipidaemic participants with impaired fasting glucose and which showed that rosuvastatin dose-dependently decreased insulin sensitivity and increased insulin secretion [3]. The authors speculated that compensatory hyperinsulinaemia induced by rosuvastatin prevented the conversion to diabetes. However, insulin secretion was not adjusted for insulin sensitivity, and therefore their study is inconclusive with respect to the interpretation of hyperinsulinaemia.

We analysed insulin sensitivity among Metabolic Syndrome in Men (METSIM) participants on rosuvastatin treatment $(n=181)$ compared with participants who were not on

Markku Laakso

markku.laakso@kuh.fi

1 Institute of Clinical Medicine, Internal Medicine, University of Eastern Finland and Kuopio University Hospital, P.O. Box 1627, 70211 Kuopio, Finland statin therapy $(n=6,569)$ [2]. We found that rosuvastatin decreased insulin sensitivity, evaluated with the Matsuda Index of Insulin Sensitivity (Matsuda ISI), by $22.2 \%$, which is of similar magnitude to simvastatin $(21.9 \%)$ and atorvastatin (24.4\%) [Table 3, reference 2]. Our results do not support the conclusion of Dr Kostapanos and collaborators that insulin resistance is particularly prominent in individuals on rosuvastatin treatment [3]. In addition, we evaluated insulin secretion by the Disposition Index (DI), which is a marker of insulin secretion adjusted for Matsuda ISI [2]. Rosuvastatin treatment decreased insulin secretion by $7.1 \%$, which is very similar to decreases of insulin secretion by simvastatin $(7.6 \%)$ and atorvastatin (7.4\%) [2]. Therefore, our study indicates that rosuvastatin treatment, indeed, impairs insulin secretion, and the hyperinsulinaemia observed in the study by Kostapanos and collaborators [3] is very likely to be caused by the lack of adjustment for prevailing insulin sensitivity.

We also compared the changes in insulin sensitivity and insulin secretion according to different doses of rosuvastatin, simvastatin and atorvastatin. Rosuvastatin dose of 5 or $10 \mathrm{mg} /$ day (only three participants had the lowest dose of $5 \mathrm{mg}$ /day) decreased insulin sensitivity by $19.6 \%$, a very similar decrease to that of low dose (10 or $20 \mathrm{mg}$ /day) simvastatin (20.8\%) and low dose (10 mg/day) atorvastatin (16.6\%). A high dose rosuvastatin (20 or $40 \mathrm{mg} /$ day) decreased insulin sensitivity by $28.6 \%$, a high dose simvastatin (40 or $80 \mathrm{mg} /$ day) by $25.4 \%$, and a high dose atorvastatin (20 or $40 \mathrm{mg} /$ day) by $30.2 \%$. Insulin secretion decreased on low and high dose rosuvastatin treatments by $4.7 \%$ and $13.6 \%$, respectively. The corresponding decreases for low and high dose simvastatin treatments were $6.6 \%$ and $9.8 \%$, respectively, and for low and high dose atorvastatin treatments were $3.4 \%$ and $10.5 \%$, respectively. These results show that rosuvastatin decreased both insulin sensitivity and insulin secretion in a dose-response manner similarly to simvastatin and atorvastatin. Thus, our 
study does not support the conclusion by Kostapanos and collaborators that statins reduce insulin sensitivity but improve insulin secretion [3].

Rosuvastatin is hydrophilic, in contrast to simvastatin and atorvastatin, which are lipophilic. Dr Kostapanos and collaborators have speculated that this property of statins is essential in the development of statin-induced diabetes [4]. A recent meta-analysis [5] and a cohort study including 239,628 individuals [6] showed that rosuvastatin increased the risk of diabetes by $17 \%$ and $42 \%$, respectively, whereas simvastatin increased the risk of diabetes by $10 \%$ and $14 \%$, respectively, and atorvastatin by $30 \%$ and $25 \%$, respectively. Pravastatin increased the risk of diabetes in these two studies by only $3 \%$ and $2 \%$, respectively $[5,6]$. In addition, a recent study demonstrated that the risk of incident diabetes was increased with atorvastatin by $22 \%$, rosuvastatin by $18 \%$ and simvastatin by $10 \%$, compared with pravastatin treatment [7]. Thus, the risk of diabetes is considerably different between the two hydrophilic statins, pravastatin and rosuvastatin, and it is unlikely that properties of different statins (hydrophilic/lipophilic) could explain differences in the risk of diabetes among the users of statin therapy. However, hydroxy-methyl-glutaryl coenzyme A (HMG-CoA) reductase inhibition could at least in part explain the differences between the statins. A recent study including 223,463 individuals demonstrated that a single nucleotide polymorphism of the HMGCR gene, encoding HMG$\mathrm{CoA}$ reductase, was associated with a $2 \%$ increased risk for type 2 diabetes [8].

Finally, we compared the effects of all hydrophilic (pravastatin, rosuvastatin, $n=209$ ) and all lipophilic statins (atorvastatin, simvastatin, lovastatin, fluvastatin, $n=1,916$ ) on insulin sensitivity and insulin secretion in the METSIM Study. Insulin sensitivity did not differ between participants on hydrophilic (5.54 \pm 3.09 , mean $\pm \mathrm{SD})$ and lipophilic statins (5.68 \pm 3.42 , $p=0.989)$. Similarly, insulin secretion was not different between METSIM participants who were on hydrophilic statins (152.56 \pm 59.61$)$ and lipophilic statins (154.32 \pm 67.21 , $p=0.911$ ). Therefore, our study does not support the view that hydrophilic and lipophilic statins differ in their effects on insulin sensitivity and insulin secretion [2].

In conclusion, our findings from the METSIM Study show that rosuvastatin does not essentially differ from other statins with respect to its effects on insulin sensitivity and insulin secretion.

Funding This work has been supported by the Academy of Finland, the Finnish Diabetes Research Foundation, the Finnish Cardiovascular Research Foundation, the Strategic Research Funding from the University of Eastern Finland, Kuopio, Finland, and EVO grant 5263 from the Kuopio University Hospital. The funding sources did not have any role in the study design; in the collection, analysis and interpretation of data; in the writing of the report; and in the decision to submit the paper for publication.

Duality of interest The authors declare that there is no duality of interest associated with this manuscript.

Contribution statement All authors were responsible for drafting the article and revising it critically for important intellectual content. All authors approved the version to be published.

\section{References}

1. Kostapanos MS, Agouridis AP, Elisaf MS (2015) Variable effects of statins on glucose homeostasis parameters and their diabetogenic role. Diabetologia. doi:10.1007/s00125-015-3633-5

2. Cederberg H, Stančáková A, Yaluri N, Modi S, Kuusisto J, Laakso $M$ (2015) Increased risk of diabetes with statin treatment is associated with impaired insulin sensitivity and insulin secretion: a 6 year follow-up study of the METSIM cohort. Diabetologia 58:1109-1117

3. Kostapanos MS, Milionis HJ, Agouridis AD, Rizos CV, Elisaf MS (2009) Rosuvastatin treatment is associated with an increase in insulin resistance in hyperlipidaemic patients with impaired fasting glucose. Int J Clin Pract 63:1308-1313

4. Kostapanos MS, Liamis GL, Milionis HJ, Elisaf MS (2010) Do statins beneficially or adversely affect glucose homeostasis? Curr Vasc Pharmacol 8:612-631

5. Preiss D, Sattar N (2012) Pharmacotherapy: statins and new-onset diabetes - the important questions. Nat Rev Cardiol 9:190-192

6. Zaharan NL, Williams D, Bennett K (2013) Statins and risk of treated incident diabetes in a primary care population. Br J Clin Pharmacol 75:1118-1124

7. Carter AA, Gomes T, Camacho X, Juurlink DN, Shah BR, Mamdani MM (2013) Risk of incident diabetes among patients treated with statins: population based study. BMJ 346:f2610

8. Swerdlow DI, Preiss D, Kuchenbaecker KB et al (2015) HMGcoenzyme A reductase inhibition, type 2 diabetes, and bodyweight: evidence from genetic analysis and randomised trials. Lancet 385 : $351-361$ 